\title{
A Curvature-Corrected Low-Voltage Bandgap Reference
}

\author{
Made Gunawan, Gerard C. M. Meijer, Jeroen Fonderie, and Johan H. Huijsing, Senior Member, IEEE
}

\begin{abstract}
A new curvature-corrected bandgap reference is presented which is able to function at supply voltages as low as $1 \mathrm{~V}$, at a supply current of only $100 \mu \mathrm{A}$. After trimming this bandgap reference has a temperature coefficient (TC) of $\pm 4 \mathrm{ppm} /{ }^{\circ} \mathrm{C}$. The reference voltage is about $200 \mathrm{mV}$ and it can simply be adjusted to higher reference voltages. The temperature range of this circuit is from 0 to $125^{\circ} \mathrm{C}$. This bandgap reference is realized using a standard bipolar process with base-diffused resistors.
\end{abstract}

\section{INTRODUCTION}

$\mathbf{V}$ OLTAGE references are used in many types of analog circuits for signal processing, such as A-D converters, smart sensors, D-A converters, etc. Of all the types of references, only bandgap references are suited to operate at very low supply voltages.

In a bandgap reference, the reference voltage is obtained by compensating the base-emitter voltage of a bipolar transistor $\left(V_{b e}\right)$ for its temperature dependence. The temperature dependence of the base-emitter voltage of a transistor, biased by a proportional-to-absolute-temperature (PTAT) current, can be represented by the following equation [1]:

$$
V_{b e}(T)=V_{g 0}-\frac{V_{g 0}-V_{b e}\left(T_{R}\right)}{T_{R}} T-(\eta-1) \frac{k T}{q} \ln \left(\frac{T}{T_{R}}\right)
$$

where $V_{g 0}$ is the extrapolated bandgap voltage at $0 \mathrm{~K}, V_{b e}\left(T_{R}\right)$ is the base-emitter voltage at the reference temperature $T_{R}$, and $\eta$ is a process-dependent constant. Practical values for these parameters are $V_{g 0}=1170 \mathrm{mV}, V_{b e}\left(T_{R}\right)=0.65 \mathrm{~V}$, $T_{R}=300 \mathrm{~K}$, and $\eta=3.6$.

The existing bipolar bandgap references operate generally at a supply voltage larger than $1 \mathrm{~V}$. The only reference known to the authors that is able to operate at a supply voltage of $1 \mathrm{~V}$ is the one introduced by Widlar [2].

In this paper, a low-voltage bandgap reference with a lower temperature coefficient (TC) than that of Widlar's circuit is

Manuscript received September 8, 1992; revised February 17, 1993.

M. Gunawan was with the Department of Electrical Engineering, Delft University of Technology, $2628 \mathrm{CD}$ Delft, The Netherlands. He is now with the Indonesian Government.

G. C. M. Meijer is with the Laboratory of Electronics, Department of Electrical Engineering, Delft University of Technology, 2628 CD Delft, The Netherlands.

J. Fonderie was with the Laboratory of Electronic Instrumentation, Department of Electrical Engineering, Delft University of Technology $2628 \mathrm{CD}$ Delft, The Netherlands. He is now with Philips Semiconductors, Sunnyvale, CA 94088 .

J. H. Huijsing is with the Laboratory of Electronic Instrumentation, Department of Electrical Engineering, Delft University of Technology, 2628 CD Delft, The Netherlands.

IEEE Log Number 9209023.

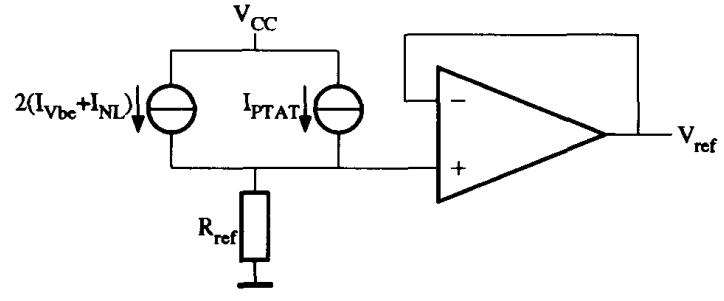

Fig. 1. The principle of the low-voltage bandgap reference.

discussed. This low TC is achieved by a new method for the compensation of the nonlinear temperature dependence (curvature correction) of $V_{b e}$. Further, within a certain range the bandgap reference can simply be adjusted for other references voltages.

\section{PRINCIPLE AND IMPLEMENTATION OF THE LOW-VOLTAGE BANDGAP REFERENCE}

The principle of the low-voltage bandgap reference is shown in Fig. 1. A current proportional to $V_{b e}\left(2 I_{V b e}\right)$ and a nonlinear correction current $\left(2 I_{N L}\right)$ are generated. When the nonlinear (curvature) correction is performed correctly, $2\left(I_{V b e}+I_{N L}\right)$ should consist of a constant component and a component that is PTAT. This latter component can be compensated by using a PTAT current source. The sum of the currents is converted into the voltage reference by using a resistor $\left(R_{\text {ref }}\right)$. The buffer circuit is applied to obtain a sufficiently low output impedance.

For the PTAT current source, the circuit shown in Fig. 2 is used [3], where the emitter-area ratio of $Q_{1}$ and $Q_{2}$ amounts to 4 .

The magnitude of the PTAT current is given by

$$
I_{\mathrm{PTAT}}=\frac{k T}{q R_{1}} \ln 4
$$

The capacitor $C_{m 1}$ is required to obtain high-frequency stability of the circuit. The transistor $Q_{5}$ is biased at a current which is twice as large as those of $Q_{1}$ and $Q_{2}$ in order to obtain full compensation of the effect of the base currents.

The new circuit generating $I_{V b e}$ as well as the curvaturecorrection current $I_{N L}$ is shown in Fig. 3. The PTAT currents in Fig. 3 are generated by the circuit shown in Fig. 2. The current $I_{V b e}=V_{b e 3} / R_{2}$ is derived from the base-emitter voltage of $Q_{3}$. The internal feedback in the circuit ensures that biasing at the indicated current levels is obtained. The transistors $Q_{3}$ and $Q_{4}$, which have an emitter-area ratio $1: p$, 


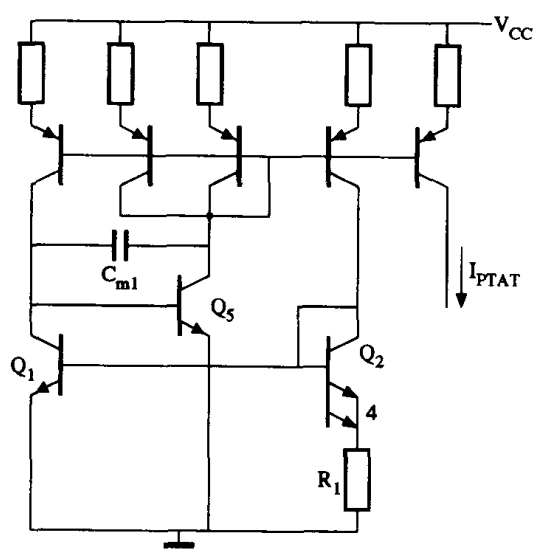

Fig. 2. The circuit generating the PTAT current.

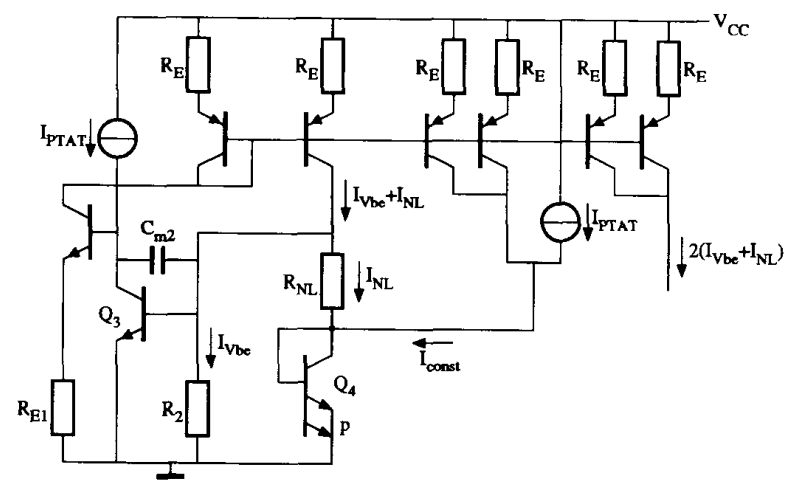

Fig. 3. The circuit generating the currents $I_{\mathrm{V} b e}$ and $I_{N L}$.

are driven by two different currents, $I_{\mathrm{PTAT}}$ and $I_{\text {const }}+I_{N L}$, respectively. The voltage across $R_{N L}$ equals the difference $\Delta V_{b e}$ of the base-emitter voltages of $Q_{3}$ and $Q_{4}$, so that

$$
\begin{aligned}
I_{N L} & =\frac{k T}{q R_{N L}} \ln \left(\frac{I_{C 3}}{I_{C 4}} \frac{I_{S 4}}{I_{S 3}}\right) \\
& =\frac{k T}{q R_{N L}} \ln \left(p \frac{I_{\mathrm{PTAT}}}{I_{\text {const }}+I_{N L}}\right)
\end{aligned}
$$

where $I_{S 3}$ and $I_{S 4}$ represent the saturation currents of $Q_{3}$ and $Q_{4}$, respectively, and where it is supposed that $p I_{S 3}=I_{S 4}$.

The resistor $R_{N L}$ is chosen in such a way that the temperature-dependent part of the current $2\left(I_{V b e}+I_{N L}\right)$ and I PTAT compensate each other and a temperature-independent current $I_{\text {const }}$ (Fig. 3) is obtained for which it holds that

$$
I_{\text {const }}=2\left(I_{V b e}+I_{N L}\right)+I_{\mathrm{PTAT}}=\frac{2 V_{g 0}}{R_{2}} .
$$

Using (1), for the current through the resistor $R_{2}$ it is found that

$$
\begin{aligned}
I_{V b e}(T)= & \frac{V_{b e 3}}{R_{2}}=\frac{V_{g 0}}{R_{2}}-\frac{V_{g 0}-V_{b e 3}\left(T_{R}\right)}{R_{2} T_{r}} T \\
& -(\eta-1) \frac{k T}{q R_{2}} \ln \frac{T}{T_{R}} .
\end{aligned}
$$

TABLE I

SPECIFICATION OF THE LOW-VOLTAGe BandGap REFERENCE $V_{C C}=1 \mathrm{~V}$, AND $R_{L}=100 \mathrm{k} \Omega$

\begin{tabular}{c|c|c|c}
\hline Parameter & Simulation Result & Measurement result & Units \\
\hline \hline Output Voltage & 195 & 199.2 & $\mathrm{mV}$ \\
\hline $\begin{array}{c}\text { Temp. Coefficient } \\
\text { orer the temperature range } \\
\text { from 0 to 125 }\end{array}$ & \pm 4 & \pm 3 & $\mathrm{ppm} /{ }^{\circ} \mathrm{C}$ \\
\hline $\begin{array}{c}\text { Line Regulation } \\
\text { Output Resistance }\end{array}$ & 100 & -450 & $\mathrm{ppm} V_{\text {ost }} / V_{C C}$ \\
\hline $\begin{array}{c}\text { Total Supply Current } \\
\text { Supply Voltage Range }\end{array}$ & $1-10$ & 150 & $\Omega$ \\
\hline $\begin{array}{c}\text { Noise } \\
\text { Temperature Range }\end{array}$ & $0-125$ & $1-2$ & $\mathrm{NA}$ \\
\hline $\begin{array}{c}\text { from 0 to 125 } \\
\text { over }\end{array}$ & $<100$ & $0-125$ & $\mathrm{~V}$ \\
\hline
\end{tabular}

The capacitor $C_{m 2}$ and the resistor $R_{E 1}$ provide the stability of the circuit.

The current $I_{V b e}$ has a component which is proportional to $T$ as represented by the second term of (5). The double value of this component is compensated by the PTAT current (see (4)), which, when using (2) and (5), results in the condition

$$
\frac{R_{2}}{R_{1}}=\frac{2\left(V_{g 0}-V_{b e 3}\left(T_{R}\right)\right)}{\frac{k T_{R}}{q} \ln 4} .
$$

To obtain the curvature correction, the magnitude of $I_{N L}$ (see (3)) has to be equal to the magnitude of the third, nonlinear, term of (5), which approximately results in the following two conditions:

$$
\frac{R_{2}}{R_{N L}} \simeq(\eta-1)
$$

and

$$
p \simeq \frac{V_{g 0}}{V_{g 0}-V_{b e 3}\left(T_{R}\right)} .
$$

Substitution of the values of $p$ and $R_{2} / R_{N L}$ in (3), (5), and (4), successively, shows that there still rests a minor nonlinear term $I_{N L}^{\prime}$. For this residual term it can be derived that

$$
\begin{aligned}
I_{N L}^{\prime} & =-\frac{k T}{q R_{N L}} \ln \left(1+\frac{I_{N L}}{I_{\text {const }}}\right) \\
& \simeq\left(\frac{k T}{q R_{N L}}\right)^{2} \frac{{ }^{2} \ln \left(p \frac{I_{\mathrm{PTAT}}}{I_{\text {const }}}\right)}{I_{\text {const }}} .
\end{aligned}
$$

Further, the TC of the resistors causes some nonlinearity, which can be explained as follows. All of the currents are 


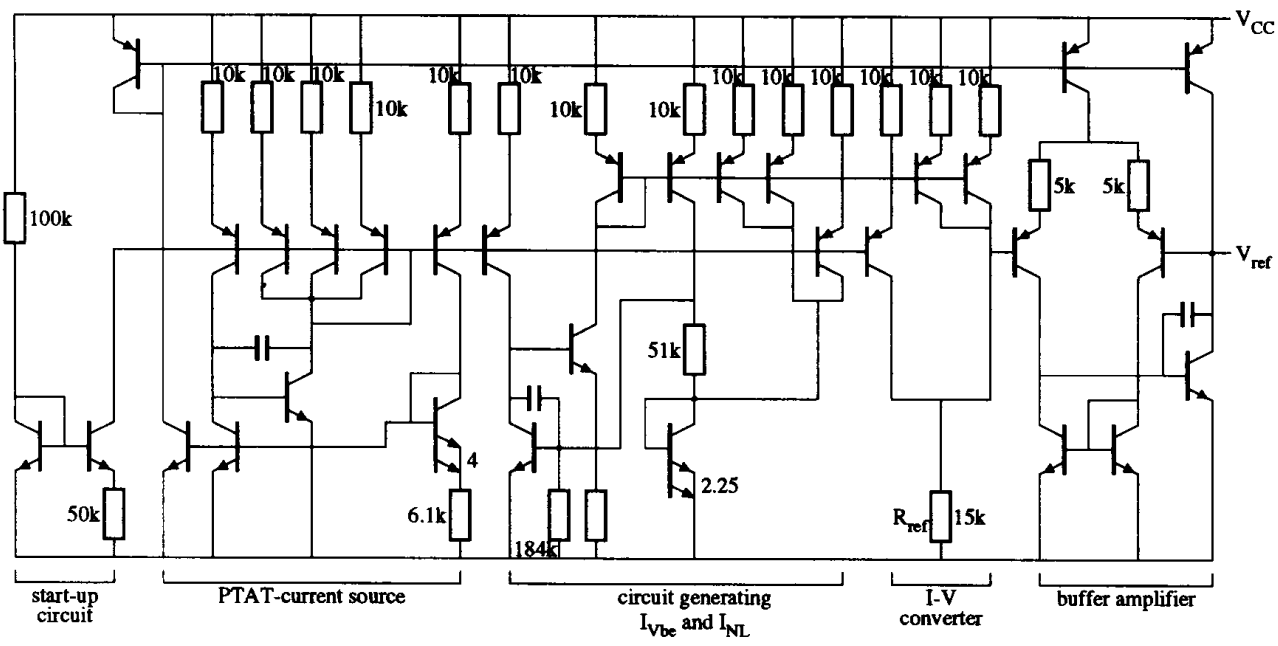

Fig. 4. The complete circuit of the low-voltage bandgap reference.

derived from the basic voltage $V_{b e}$ and $V_{\mathrm{PTAT}}$ and converted to the output voltage $V_{\text {ref }}$. The conversion factors in this process of signal conversion are obtained with resistors, and determined by resistor ratios.

When well-matched resistors are used these ratios are independent of temperature. However, the basic voltage $V_{b e 3}$ is also affected by the resistor TC's, because this TC causes the biasing current $I_{\text {PTAT }}$ to be not really PTAT.

In [4] Meijer shows that this effect causes a small nonlinearity, which is compensated for when the condition (7) is modified into

$$
\left.\frac{R_{2}}{R_{N L}}\right|_{T C}=\eta-1+2 \alpha_{1} T_{R}
$$

where $\alpha_{1}$ is the first-order (linear) component of the TC. In our design the empirical values $\eta=3.5$ and $\alpha_{1}=1700 \mathrm{ppm} /{ }^{\circ} \mathrm{C}$ have been used to calculate the resistor ratio $R_{2} / R_{N L}$. Finally, this ratio has been further optimized to compensate for the residual term $I_{N L}^{\prime}$ of (9).

The results of this analysis are found to be in good agreement with numerical ones obtained using the SPICE computer program. The SPICE simulation results are listed in Table I.

\section{Measurement Results and Discussion}

The complete circuit is shown in Fig. 4. The start-up circuit generates a small current which is injected into the bases of the $\mathrm{p}-\mathrm{n}$ - $\mathrm{p}$ current mirror and drives the circuit towards the desired state of biasing.

This complete circuit is integrated using a standard bipolar process with base-diffused resistors. The circuit is calibrated for the nominal value of $V_{\text {ref }}$ by trimming the resistor $R_{2}$ using a fusible-link trimming method [1]. For $V_{\text {ref }}$ it holds that

$$
V_{\text {ref }}=2 \frac{R_{\text {ref }}}{R_{2}} V_{g 0}
$$

Provided that the resistor ratio $R_{\text {ref }} / R_{2}$ is a well-known constant which is completely predetermined by the layout, then

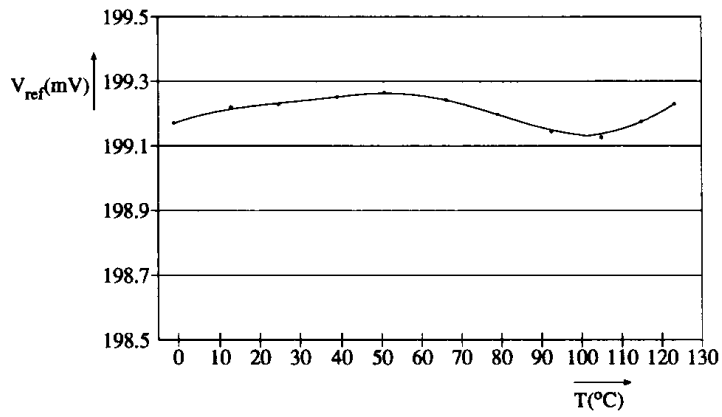

Fig. 5. Measured value of $V_{\text {ref }}(T)$ at a supply voltage of $1 \mathrm{~V}$.

this low-voltage reference has the typical attractive feature of bandgap references that the output voltage is temperatureindependent when this voltage is adjusted for a predetermined value, where also the (PTAT) offset voltage of output buffer amplifier and the mismatches of the current-mirror transistors are compensated for.

In practice, a slight deviation for the optimal value of $V_{\text {ref }}$ (i.e., the value with the lowest TC) is found (see Table I). This deviation is mainly attributable to the effect of the (nonlinear) $\mathrm{TC}$ of the resistors.

The temperature dependency of the output voltage is shown in Fig. 5, which depicts the measurement results for a supply voltage of $1 \mathrm{~V}$. This measurement result agrees with the values found by simulation. Other measurement results are listed in Table I. The measured line regulation differs considerably from what was expected from the simulations. This effect is caused by the voltage dependency of the base-diffused resistors, particularly the resistors $R_{1}, R_{2}$, and $R_{N L}$.

A base-diffused resistor is implemented in an epitaxial island. This island has to be biased by a certain voltage, in this case the positive supply voltage. The magnitude of the resistor is affected by the voltage between the epitaxial island and the resistor $\left(V_{\text {epi-sp }}\right)$. The voltage dependency affects the performance of the circuit because the resistors $R_{1}$ and 
$R_{2}$ have a $V_{\text {epi-sp }}$ that is one diode voltage higher than the $V_{\text {epi-sp }}$ of resistor $R_{N L}$. It was found that when the supply voltage increases, the magnitude of $R_{N L}$ increases more than the magnitude of $R_{1}$ and $R_{2}$. This problem can easily be solved by placing a forward-biased diode between the epi contact and the bias voltage of $R_{1}$ and $R_{2}$ so that $V_{\text {epi-sp }}$ of $R_{1}, R_{2}$, and $R_{N L}$ all have the same value.

In view of this effect during the test, the voltage range has been limited to the $1-2-\mathrm{V}$ range.

\section{CONCLUSION}

The realized circuit mainly shows the performance as expected, based on simulations. The minimum supply voltage is $1 \mathrm{~V}$ for an operating temperature range from 0 to $125^{\circ} \mathrm{C}$. The circuit also operates at temperatures lower than $0^{\circ} \mathrm{C}$, but then a slightly higher supply voltage has to be tolerated. Also the curvature-correction circuit functions as expected. The only difference in the performance of the circuit emanates from the voltage dependency of the base-diffused resistors of the standard bipolar process used. This effect can, however, be eliminated, as was explained.

\section{ACKNOWLEDGMENT}

The authors wish to acknowledge the Delft Institute of Microelectronics and Submicron Technology (DIMES) for processing the chips.

\section{REFERENCES}

[1] G. C. M. Meijer, "Thermal sensors based on transistors," Sensors and Actuators, vol. 10, pp. 103-125, 1986.

[2] R. J. Widlar, "A new breed of lineair ICs runs at 1-volt levels," Electronics, pp. 115-199, Mar. 29, 1979.

[3] H. C. Nauta and E. H. Nordholt, "A new class of high performance PTAT current sources," Electron. Lett., vol. 21, pp. 384-386, 1985.

[4] G. C. M. Meijer, "A low power easy-to-calibrate temperature transducer," IEEE J. Solid-State Circuits, vol. SC-17, pp. 609-613, 1982.

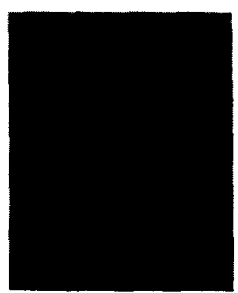

Made Gunawan was born in Denpasar, Indonesia on November 18, 1963. He received the M.Sc. degree in electrical engineering from the Delft University of Technology. The Netherlands, in 1992.

Since 1991 he has been employed by the Indonesian Government.

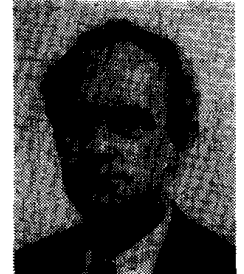

Gerard C. M. Meijer was born in Wateringen The Netherlands, on June 28, 1945. He received the ingenieurs (M.Sc.) and Ph.D. degrees in electrica engineering from the Delft University of Technology, Delft, The Netherlands, in 1972 and 1982 respectively.

Since 1972 he has been part of the Laboratory of Electronics, Delft University of Technology, where he is an Associate Professor, engaged in research and teaching on analog IC's. In 1984 and part-time during 1985-1987 he was seconded to the Delft Instruments Company in Delft where he was involved in the development of industrial-level gauges and temperature transducers.

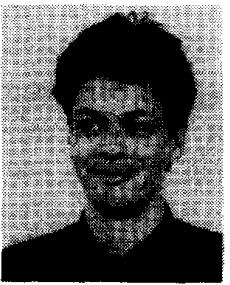

Jeroen Fonderie was bom in Amsterdam, The Netherlands, on July 27, 1960 . He received the M.Sc. degree in electrical engineering from the Delf University of Technology, Delft, The Netherlands, in 1987 and the Ph.D. degree from the same university in 1991.

From 1987 he has been a Research Assistant at the Electronic Instrumentation Laboratory, Department of Electrical Engineering, Delft University of Technology, where he has been working on the subject of low-voltage operational amplifiers. Since 1992 he has been a Senior Designer at Philips Semiconductors, Sunnyvale CA.

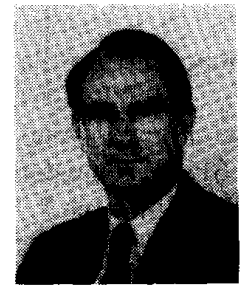

Johan H. Huijsing (SM'81) was born in Bandung, Indonesia, on May 21, 1938. He received the ingenieurs (M.Sc.) degree in electrical engineering from the Delft University of Technology, Delft, The Netherlands, in 1969, and the Ph.D. degree from the same university in 1981 for work on operational amplifiers.

Since 1969 he has been a member of the Research and Teaching Staff of the Electronic Instrumentation Laboratory, Department of Electrical Engineering, Delft University of Technology, as a full Professor. He is engaged in research on analog integrated circuits and integrated sensors for instrumentation systems. 